\title{
Integrated Transportation Network Design Optimization
}

\author{
Christine Taylor* and Olivier L. de Weck ${ }^{\dagger}$ \\ Massachusetts Institute of Technology, Cambridge, MA 02139
}

\begin{abstract}
Traditionally, the design of a transportation system has focused on one of the two systems that comprise the transportation architecture, the vehicle or the network design. However, to define a systems level architecture for a transportation system, it is necessary to expand the system definition during the design process to include the network definition, the vehicle architecture, and the operations, which couple the vehicle and the network. In this paper, the transportation architecture is decomposed into these fundamental sub-systems by classifying the decisions required to define each sub-system. Utilizing the integrated transportation system formulation, the design of the transportation architecture can be obtained by concurrently optimizing the vehicle and network decisions. By formulating the problem in this manner, the optimization can exploit the coupling between the vehicle and network definition to find a system-level solution that best satisfies the system objective. The case study presented in this paper focuses on the design of an aircraft transportation network for overnight package delivery. By concurrently optimizing both the vehicle and network definition for a selected few cities with a fixed demand, we obtain a minimum of a ten percent improvement in cost over that obtained by optimizing the network design using a set of pre-defined aircrafts. Additionally, by comparing the concurrently optimized solutions with the solutions obtained by traditional vehicle optimization, a minimum of an eleven percent improvement in cost is realized. The improvement in system cost obtained by the integrated transportation optimization implementation can be attributed to the reduction in operational inefficiencies for the transportation system.
\end{abstract}

\section{Nomenclature}

$r \quad$ Range (nmi)

$V_{c} \quad$ Velocity (kts)

$T / W$ Thrust to Weight

$n_{i k} \quad$ Number of aircraft on route $(\mathrm{i}, \mathrm{k})$

$f \quad$ Fixed cost of allocating aircraft $(\$ /$ day $)$
C Capacity (lbs)

$W / S \quad$ Wing Loading $\left(l b / f t^{2}\right)$

$N_{\text {eng }} \quad$ Number of Engines

$x_{i j k} \quad$ Number of packages on route $(\mathrm{i}, \mathrm{j}, \mathrm{k})$

$m \quad$ Variable cost of utilizing aircraft $(\$ / \mathrm{hr})$

(i,k) Aircraft route that starts at node $i$ travels to node $k$ and returns to node $i$

$(\mathrm{i}, \mathrm{j}, \mathrm{k})$ Package route that starts at node $i$ travels through node $k$ and terminates at node $j$

\section{Introduction}

$\mathrm{T}$ HE system of systems philosophy dictates an expansion of a traditional design methodology to encompass an integrated view of a system during the design process. Traditional approaches to sub-system design focus on a particular discipline and analyze the best design given sub-system performance targets. The sub-system objectives are selected to mimic the system objective; however, since the true system objective is unavailable the resulting design can be sub-optimal from the system perspective.

\footnotetext{
*Graduate Student, Department of Aeronautics and Astronautics, 77 Massachusetts Avenue, Room 33-409, c_taylor@mit.edu, AIAA Student Member

$\dagger$ Assistant Professor, Department of Aeronautics and Astronautics, Engineering Systems Division, 77 Massachusetts Avenue, Room 33-412, deweck@mit.edu, AIAA Senior Member
} 
As we expand the definition of the system, we effectively enlarge the design control volume, which defines the boundary of inputs and outputs of the system. The interior of the control volume is the design space under consideration, where the designer can manipulate the components to achieve desired outputs, given the inherent physical constraints and the external constraints input across the boundary. As the control volume expands, greater flexibility in decisions is achieved, but with this flexibility comes an increase in problem size and complexity.

In order to analyze a system in a holistic fashion we must consider where to construct the control volume. Traditionally, even in systems engineering the design boundary has been limited to the vehicle design. However for transportation systems, it is not simply the design of a single vehicle, but the interaction of multiple vehicles to achieve the desired mission objectives. By expanding the system definition to include not only the vehicle designs but the network the vehicles travel through and the operations they perform, we can obtain a true system perspective of a transportation network.

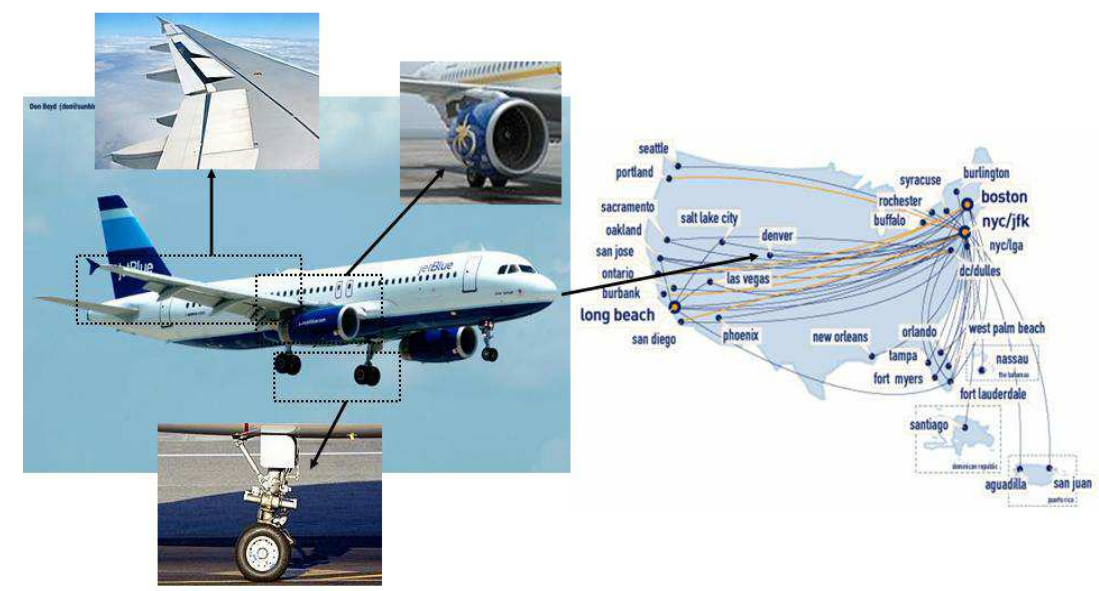

Figure 1. On the left, an Airbus 320 with specific sub-systems defined in greater detail as inserts. On the right, the Jet Blue air transportation network.

Figure 1 depicts both an aircraft with sub-system components and an air transportation network. The control volume for vehicle design can be limited to any single sub-system, a limited interaction of sub-systems, or the entire vehicle design. Similarly, network optimization theory limits the control volume to encompass only the transportation network, with the vehicle design as inputs to the problem. This paper focuses on the concurrent optimization of the aircraft and network design, effectively enlarging the control volume to include all of Figure 1 .

Reference 1 defines a system-of-systems by the level of operational independence of the components of the system. In air transportation networks, the the aircraft design and the network design are traditionally performed independently of each other and once defined each aircraft is operated independently. However, the strong coupling between the aircraft and the network provides an opportunity to improve the transportation system such that the operations are more efficiently defined.

Traditionally, the design of a transportation network focuses on determining the optimal set of operations for a given vehicle or set of vehicles such that the prescribed demand is satisfied. In Reference 2, the optimal allocation of a set of vehicles for an overnight package delivery system is considered. Given a set of vehicles with different ranges, capacities, and costs, the objective is to minimize the total network costs by choosing the routes through the network and allocating the appropriate vehicles to meet the given package demand. The example of the overnight package delivery system presented in this paper provides an excellent starting point to examine the effect of concurrently optimizing the vehicle and network characteristics, and will be 
used as the example in this work.

The transportation network design problem, and its variants, have been well researched within the operations research community. For example in Reference 3, the definition of the transportation network is presented as well as many of the resulting problems of study, such as traffic modeling, and vehicle allocation. In Reference 4 transportation network modeling was utilized to solve a school bus routing problem, where the primary constraints focused on the timing restrictions inherent in school bus pick-ups and drop-offs.

Another area of research pursued by the operations research community focuses on the allocation and scheduling of vehicles to pre-defined routes through a network. In Reference 5 the fleet assignment problem is solved in conjunction with the aircraft routing problem. By concurrently optimizing these two classes of problems, the optimal allocation of fleets to routes and the timing of each flight is determined to provide a more robust methodology for defining the flight scheduling for an airline.

Recently, investigations into the design of vehicles to fulfill multiple operations have been considered to understand the impact on the vehicle design characteristics. One such example is examined in Reference 6 . In this paper, the operations, namely the routes are specified, each with a different distance and demand. The objective is to determine the best vehicle design that satisfies the multiple operational criteria. The resulting aircraft design is not optimal for a single route, but is the best compromise in range and capacity for the system as a whole. Other investigations into the design of a vehicle to fulfill multiple operations can be found in the design of extensible spacecraft. For example, the design of a multi-function orbit transfer vehicle (OTV), as presented in Reference 7, considers the benefit of designing a single OTV bus that not only fulfills the current mission requirements but has the flexibility to be extended for potential future mission objectives.

In transportation systems, there exists a high degree of coupling between the vehicle capabilities and the network definition, as realized in the definition of the operations. When designing a complex transportation system, such as an aircraft transportation network, it is beneficial to define the system boundary to include the network definition as well as the operations into the vehicle design optimization. This expansion of the system definition allows for the coupling between the vehicle design and network definition to be exploited, thereby reducing inefficiencies in the transportation system and resulting in the optimal transportation architecture.

This paper investigates the benefits of optimizing an integrated air transportation network design where the vehicle design, network design and operations are concurrently defined. In Section II, a decomposition of the air transportation system is presented that segments the problem into the four components of the network, vehicle, operations and objective and presents the model formulation for each. Section III presents the examples analyzed in this paper and formulates the traditional design approach for network optimization and vehicle optimization, as well as the results obtained for these analyses, which will provide a baseline of comparison for the integrated system design methodology presented in this paper. Section IV compares the results obtained from the integrated system design optimization to the results of the traditional optimization methods and analyzes the improvements in the system architecture definition obtained by the integrated optimization methodology. Section V reviews the ideas and results presented and discusses continuing work on this topic.

\section{Problem Formulation}

The example considered in this paper is an extension of the problem defined in Reference 2 where the design of an overnight package delivery network is considered. In this paper, the concurrent design of an aircraft fleet and transportation network are considered, where the network is defined by a set of cities, and the arcs connecting the cities are the straight line distances between each city-pair. Each city-pair has an associated package demand that is fixed and the demand between two cities is assumed to be symmetric. The objective for the problem is to determine the lowest cost transportation system architecture that satisfies the given demand.

The integrated transportation system design problem consists of four components: the transportation network definition, the vehicle design, the operations constraints, and the system level objective. As shown in Figure 2, the vehicle and the network are the sub-systems that determine the cost of the transportation system, and the operations define the constraints that couple them. The following sub-sections describe the models and assumptions required to define each component of the problem. 


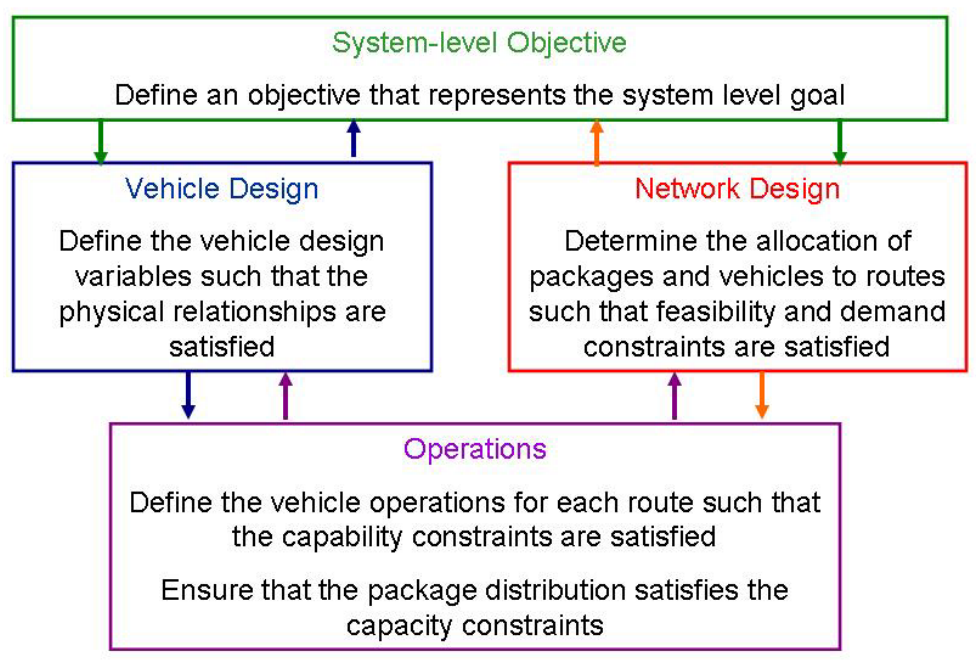

Figure 2. Diagram of the Integrated Transportation System Model

\section{A. Network Model Formulation}

The network sub-system defines the allocation of vehicles and packages to routes through the network. For the case study presented in this paper, all vehicles are assumed to fly between two cities and can perform the round trip flight once. An aircraft route is defined as $(i, k)$ where $i$ is the city the aircraft originates and terminates the path and $k$ is the destination city. The number of aircrafts flying route $(i, k)$ is defined as $n_{i k}$. Since only feasible routes are defined, the vehicle allocation constraints simply impose a limit of ten aircrafts on a given route.

The package allocation constraints ensure that the demand of each city pair is fulfilled. Although aircrafts can fly only round trips between two cities, packages may travel through an additional city towards the destination. By defining a route $(i, j, k)$ as starting at city $i$ traveling through city $k$ and terminating at city $j$, the number of packages traveling this route can be defined as $x_{i j k}$. This definition allows for packages to fly direct flights since city $k$ can be identical to city $i$ or city $j$. Therefore, $x_{i j j}$ represents the number of packages that travel from city $i$ directly to city $j$ on the first leg of an aircraft traveling on route $(i, j)$. Similarly, $x_{i j i}$ represents the number of packages that wait at city $i$ to be transferred to city $j$ on the return trip of an aircraft flying route $(j, i)$. The demand constraints that govern the feasibility of the package flow are supplied in Equation 1

$$
\sum_{k=1}^{N} x_{i j k}=P_{i j} \quad i, j=1 \ldots N
$$

Here, $P_{i j}$ is the package demand from city $i$ to city $j$, and $N$ is the total number of cities in the network.

\section{B. Vehicle Model Formulation}

The vehicle sub-system determines the performance characteristics of the vehicle, namely the range (R), capacity $(\mathrm{C})$, cruise velocity $\left(V_{c}\right)$, wing loading $(W / S)$, thrust-to-weight ratio $(T / W)$, and number of engines $\left(N_{\text {eng }}\right)$. For the purpose of this analysis, we consider a simplified model of an aircraft and calculate the takeoff weight using a model provided in Reference 8.

The weight estimate is based on a simple cruise profile, as shown in Figure 3. Each segment of the profile has an associated weight fraction that represents the ratio of the weight at the end of a segment to the weight

$$
4 \text { of } 16
$$


at the beginning of a segment. The weight ratios for take-off, climb, and decent/landing are typical values provided by Reference 8 and are listed in Table 1,

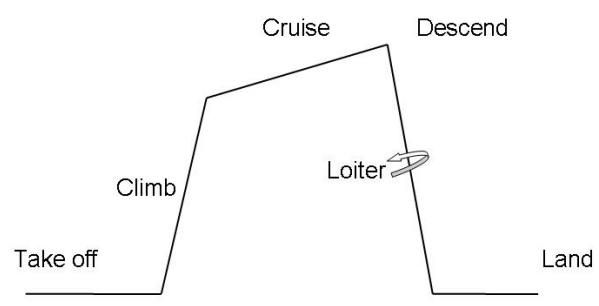

Figure 3. Diagram of a Simple Cruise Profile

Table 1. Defined Weight Ratios for Simple Cruise Profile Segments

\begin{tabular}{|l|c|}
\hline Segment & Weight Ratio \\
\hline \hline Take-off & 0.97 \\
\hline Climb & 0.985 \\
\hline Descent/Landing & 0.995 \\
\hline
\end{tabular}

The weight ratios for the cruise $\left(W_{C}\right)$ and loiter $\left(W_{L}\right)$ segments are taken from the Breguet range and endurance equations, respectively, and are listed in Equation 2.

$$
\begin{aligned}
W_{C} & =\exp \frac{-R S_{F C}}{V_{c} L / D} \\
W_{L} & =\exp \frac{-t S_{F C}}{L / D}
\end{aligned}
$$

Here, $S_{F C}$ is the specific fuel consumption of the aircraft, $L / D$ is the lift to drag ratio, and $t$ is the time spent loitering before landing. The nominal values of these parameters are listed in Table 2, By multiplying the weight ratios together, the total weight ratio $\left(W_{T}\right)$ for the entire flight profile can be estimated. The fuel fraction $\left(f_{f}\right)$ of the aircraft is computed from the total weight ratio, as shown in Equation 3, where a six percent fuel reserve is assumed.

$$
f_{f}=1.06 *\left(1-W_{T}\right)
$$

Table 2. Parameter Values for Aircraft Design

\begin{tabular}{|l|c|}
\hline Parameter & Value \\
\hline \hline$S_{F C}(1 / \mathrm{sec})$ & .6 \\
\hline $\mathrm{L} / \mathrm{D}$ & 17 \\
\hline $\mathrm{t}(\min )$ & 30 \\
\hline
\end{tabular}

The total take-off weight $\left(W_{0}\right)$ is defined to be the sum of the cargo weight, the weight of the fuel and the structural weight of the aircraft. Rearranging this relationship, we can express the total take-off weight

$$
5 \text { of } 16
$$


of the aircraft as shown in Equation 4 .

$$
W_{0}=\frac{W_{p}}{1-f_{f}-s_{f}}
$$

Here, the fuel fraction is as defined in Equation [3, and the structural fraction $\left(s_{f}\right)$ is the ratio of the structural mass over the total take-off mass. The payload weight $\left(W_{p}\right)$ is the total cargo mass of the aircraft plus the weight of the flight crew, which is assumed to consist of two people for cargo flights. For the purposes of the example presented in this paper, the cargo mass is assumed equal to the aircraft capacity (C), which decouples the aircraft performance constraints from the package distribution. The structural or dry weight of the aircraft accounts for the total unloaded and un-fueled aircraft weight and is estimated by an empirically derived formula for vehicle mass, taken from Reference 8 and shown in Equation 5 .

$$
s_{f}=1.02 W_{0}^{-.06}
$$

The total aircraft weight and the weight of the fuel are determined by numerically solving the system of equations defined by Equations 4 and 5 .

\section{Operations Model Formulation}

The operations of a transportation system determine how the vehicle performs on a given route and is defined by two sets of equations: capability and capacity constraints. The capability constraints govern the ability of a specified vehicle to travel a given path. For the aircraft transportation problem, the capability constraints are simply a constraint on the distance that an aircraft can travel without refueling. Therefore, this constraint requires that a given vehicle can not travel between two cities whose distance is greater than the range of the aircraft.

The capacity constraints determine if a given package allocation can be accommodated by the cumulative capacity of all vehicles on a given route. If we define the capacity of route $(i, k)$ as $G_{i k}$, as in Equation 6 , then the capacity constraints can be formulated as shown in Equation 7.

$$
G_{i k}=n_{i k} C
$$

$$
\begin{aligned}
& \sum_{j=1}^{N} x_{i j k} \leq G_{i k} \quad i, k=1 \ldots N \\
& \sum_{i=1}^{N} x_{i j k} \leq G_{j k} \quad j, k=1 \ldots N
\end{aligned}
$$

Since, we assume that a given vehicle travels only between two cities, the capacity of a route is the same on the return leg as it is on the outbound leg. In the case where multiple types of vehicles are defined, the capacity of a route is defined as the sum over each type of vehicle on each route and additional subscripts would be defined to distinguish the parameters for multiple vehicle designs. Finally, it is important to note that for modeling purposes the capacity and capability constraints are decoupled in this problem. Although this is not true in general, since the range of the aircraft is defined by assuming the aircraft is at maximum capacity, the actual cargo distribution would only increase the aircraft's performance on any given route.

\section{System Objective}

The system objective provides a metric for architecture evaluation. In this paper, the objective is to minimize the total system cost for a single day of operation. The aircraft has two associated cost values: a fixed cost $(f)$ that is associated with an aircraft's allocation, and a variable cost $(m)$ that is associated with an aircraft's operation. Thus, both the number of aircrafts employed and the number of hours each aircraft flies are critical for determining the total system cost.

The aircraft's performance parameters define both the fixed and variable costs for the design. The design cost models are taken from the DAPCA IV models provided in Reference 8. These relations are based on empirical data of previous aircraft designs and are dependent on parameters such as size, capability, and predicted use. Since the purpose of this paper is to present a method for transportation system design, using the cost relationships in Reference 8 provides a metric for comparison between different architectures.

$$
6 \text { of } 16
$$


The cost model uses the weight $(W)$, velocity $\left(V_{c}\right)$, number of engines $\left(N_{\text {eng }}\right)$ and thrust per engine $\left(T_{\text {eng }}\right)$ as inputs to compute the research, development, testing, and evaluation costs. These non-recurring costs set the purchase price and can be used to determine the depreciation of the aircraft. The fixed cost $(f)$ of the aircraft is the cost per day of ownership, and is equivalent to the per-day depreciation of the aircraft.

The number of hours in flight and the difficulty in maintaining the aircraft determine the operations costs. Using the relationships provided in Reference 8 an estimate for the man-hours and work-hours required to both fly and maintain the aircraft can be computed. The variable costs $(m)$ are the recurring costs associated with aircraft usage, and can therefore be computed as the cost per hour of aircraft flight.

The total system operating costs are defined as

$$
J=\sum_{i=1}^{N} \sum_{j=1}^{N} c_{i k} n_{i k}
$$

where $c_{i k}$ represents the cost of an aircraft traveling on a given route, as expressed in Equation 9 .

$$
c_{i k}=\left\{\begin{array}{ccc}
f+m \frac{2 d_{i k}}{V_{c}} & , \quad r \geq d_{i k}, \quad i \neq k \\
\infty & , \quad r<d_{i k}, \quad i \neq k \\
0 & , & i=k
\end{array}\right.
$$

Equation 9 imposes a cost equal to the fixed cost plus twice the time required to travel a single leg of the trip multiplied by the variable cost per hour of flying to an aircraft, if the aircraft can fly a given leg, as determined by the range requirement. If an aircraft does not have the range required to travel a given leg, a large cost is assigned to prohibit the selection. Finally, in the current model, storage at a given city is free, and therefore, same city 'transfers' have no cost.

\section{Transportation System Design}

The models described above are implemented for the two different cases defined in Reference 2 . The first case is a network of the first seven cities, alphabetically, of Albuquerque (ABQ), Atlanta (ATL), Boston (BOS), Charlotte (CLT), Chicago (ORD), Cincinnati (CVG), and Cleveland (CLE). The distance and demand information is provided in Tables 3 and 4, respectively. The second case is a network of the largest seven cities, in terms of demand, of Atlanta (ATL), Boston (BOS), Chicago (ORD), Dallas (DFW), Los Angeles (LAX), New York (JFK), and San Francisco (SFO). The distance and demand information is provided in Tables 5] and 6, respectively.

Table 3. City to City Distances for First Seven City Network (nautical miles)

\begin{tabular}{|l||c|c|c|c|c|c|c|}
\hline & ABQ & ATL & BOS & CLT & ORD & CVG & CLE \\
\hline \hline ABQ & 0 & 1222 & 1933 & 1426 & 1160 & 1209 & 1393 \\
\hline ATL & 1222 & 0 & 934 & 208 & 622 & 400 & 619 \\
\hline BOS & 1933 & 934 & 0 & 731 & 882 & 755 & 563 \\
\hline CLT & 1426 & 208 & 731 & 0 & 682 & 423 & 448 \\
\hline ORD & 1160 & 622 & 882 & 682 & 0 & 260 & 309 \\
\hline CVG & 1209 & 400 & 755 & 423 & 260 & 0 & 219 \\
\hline CLE & 1393 & 619 & 563 & 448 & 309 & 219 & 0 \\
\hline
\end{tabular}

For each of the networks defined, traditional optimization approaches are employed to solve the problem in order to provide a basis for comparison for the integrated optimization methodology presented in this paper. The traditional optimization methodology embodies two views: network optimization and vehicle optimization and the following sections detail the results of each analysis.

\section{A. Traditional Network Optimization}

In traditional network optimization a set of vehicles are defined, each with an associated cost and capability. Using these pre-defined vehicle parameters, an optimal allocation of vehicles to routes can be defined to meet

$$
7 \text { of } 16
$$


Table 4. Demand for First Seven City Network (lbs)

\begin{tabular}{|l||c|c|c|c|c|c|c|}
\hline & ABQ & ATL & BOS & CLT & ORD & CVG & CLE \\
\hline \hline ABQ & 0 & 2356 & 2051 & 673 & 4572 & 214 & 747 \\
\hline ATL & 2356 & 0 & 14045 & 4610 & 31313 & 1465 & 5112 \\
\hline BOS & 2051 & 14045 & 0 & 4014 & 27261 & 1276 & 4451 \\
\hline CLT & 673 & 4610 & 4014 & 0 & 8948 & 419 & 1461 \\
\hline ORD & 4572 & 31313 & 27261 & 8948 & 0 & 2844 & 9923 \\
\hline CVG & 214 & 1465 & 1276 & 419 & 2844 & 0 & 464 \\
\hline CLE & 747 & 5112 & 4451 & 1461 & 9923 & 464 & 0 \\
\hline
\end{tabular}

Table 5. City to City Distances for Largest Seven City Network (nautical miles)

\begin{tabular}{|l||c|c|c|c|c|c|c|}
\hline & ATL & BOS & ORD & DFW & LAX & JFK & SFO \\
\hline \hline ATL & 0 & 934 & 622 & 688 & 1921 & 756 & 2179 \\
\hline BOS & 934 & 0 & 882 & 1538 & 2629 & 183 & 2729 \\
\hline ORD & 622 & 882 & 0 & 806 & 1767 & 713 & 1866 \\
\hline DFW & 688 & 1538 & 806 & 0 & 1257 & 1360 & 1518 \\
\hline LAX & 1921 & 2629 & 1767 & 1257 & 0 & 2454 & 330 \\
\hline JFK & 756 & 183 & 713 & 1360 & 2454 & 0 & 2560 \\
\hline SFO & 2179 & 2729 & 1866 & 1518 & 330 & 2560 & 0 \\
\hline
\end{tabular}

Table 6. Demand for Largest Seven City Network (lbs)

\begin{tabular}{|l||c|c|c|c|c|c|c|}
\hline & ATL & BOS & ORD & DFW & LAX & JFK & SFO \\
\hline \hline ATL & 0 & 14045 & 31313 & 19984 & 34506 & 57949 & 37318 \\
\hline BOS & 14045 & 0 & 27261 & 17398 & 30041 & 50451 & 32489 \\
\hline ORD & 31313 & 27261 & 0 & 38788 & 66975 & 112479 & 72434 \\
\hline DFW & 19984 & 17398 & 38788 & 0 & 42743 & 71784 & 46227 \\
\hline LAX & 34506 & 30041 & 66975 & 42743 & 0 & 123948 & 79820 \\
\hline JFK & 57949 & 50451 & 112479 & 71784 & 123948 & 0 & 134050 \\
\hline SFO & 37318 & 32489 & 72434 & 46227 & 79820 & 134050 & 0 \\
\hline
\end{tabular}


the demand on the network. In Reference 2 three types of aircraft are considered available for use and no limit on the number of each type of aircraft is given. The three types of aircraft are chosen to provide a representative sample for a small (Plane A), medium (Plane B), and large (Plane C) plane. Using the cost calculation described above, the fixed and variable costs can be calculated from the vehicle characteristics and the relevant parameters of each aircraft are given in Table 7.

Table 7. Pre-defined Aircraft Specifications

\begin{tabular}{|l|c|c|c|}
\hline Parameter & Plane A & Plane B & Plane C \\
\hline \hline Capacity $C($ lbs $)$ & 5,000 & 72,210 & 202,100 \\
\hline Range $R($ nmi $)$ & 1,063 & 3,000 & 3,950 \\
\hline Velocity $V_{c}($ kts $)$ & 252 & 465 & 526 \\
\hline Fixed Cost $f(\$ /$ day $)$ & 1,481 & 10,616 & 26,129 \\
\hline Linear Cost $m(\$ / h r)$ & 758 & 3,116 & 7,194 \\
\hline
\end{tabular}

Using the above parameters as cost values, and the network and cost models described above, an optimal allocation of vehicles to routes can be determined by employing CPLEX as a linear, mixed-integer optimization algorithm. For the case of the first seven city network defined in Tables 3 and 4, the optimal cost is $\$ 107,888$, and the solution is depicted in Figure 4 .

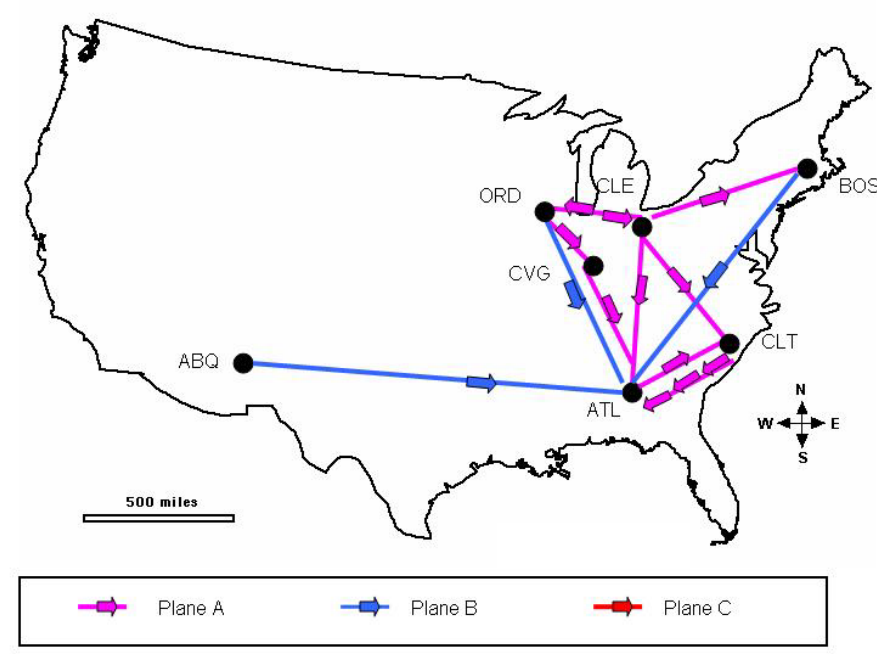

Figure 4. Optimal Allocation of Three Types of Aircraft for First Seven City Network

As shown in Figure 4, Atlanta becomes a hub in the network, with incoming flights from every other city. The hub, however, is augmented by additional flights between other cities, to lessen the package flow into and out of Atlanta, and therefore most routes can accommodate the demand using a single flight of the smallest plane, Plane A. Although the demand into and out of Albuquerque is low, requiring at most two outgoing Plane A flights, a single Plane B is allocated due to the range constraint. Since Plane B is oversized in terms of both range and capacity, for all routes that it is allocated for, there is a considerable amount of slack in this transportation system.

For the case of the seven largest city network defined in Tables 5 and 6 , the optimal cost is $\$ 517,030$ and the optimal allocation is depicted in Figure 5.

As shown in Figure 5, only the medium (Plane B) and large (Plane C) planes are allocated due to both the range and capacity constraints. For this network, Chicago becomes a hub and Dallas has incoming flights

$$
9 \text { of } 16
$$




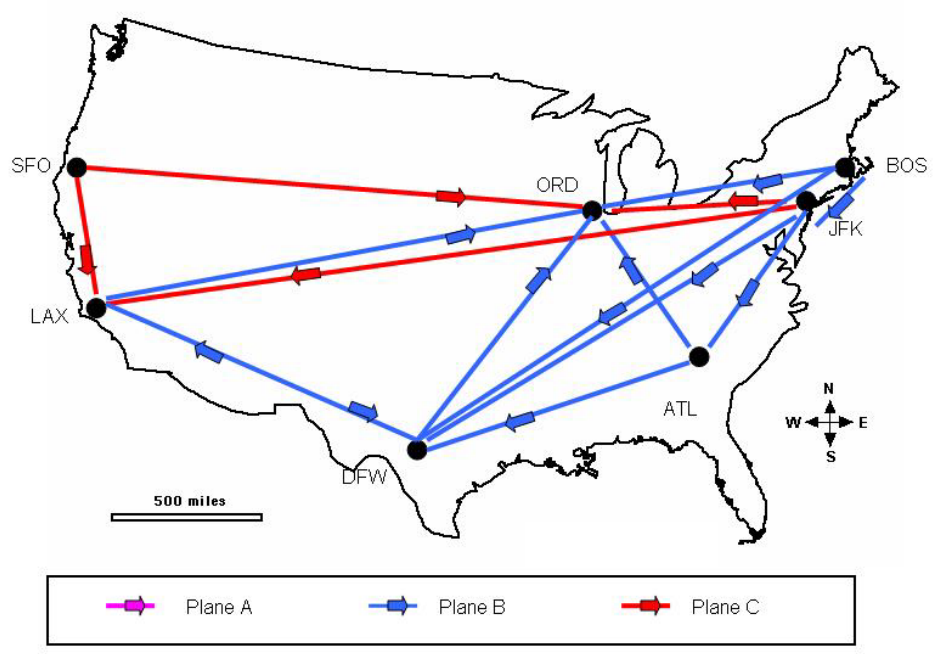

Figure 5. Optimal Allocation of Three Types of Aircraft for Largest Seven City Network

from every city except San Francisco. Thus, with this level of demand, it is necessary to utilize the largest plane for San Francisco, since it is only connected to Los Angeles and Chicago. Additionally, Plane C is utilized on the New York to Los Angeles and New York to Chicago routes to accommodate the large number of packages originating in New York.

\section{B. Traditional Vehicle Optimization}

In traditional vehicle optimization the routes are selected apriori and the vehicle design characteristics are optimized to produce the lowest system cost. For the traditional vehicle design optimization problem, a hubspoke network configuration is assumed where a single city in the network is designated as the 'hub' and all routes in the network connect to this city. More precisely, a hub is defined as a node with nodal degree $N-1$, where $N$ represents the number of nodes in the network. The optimal vehicle design characteristics defined are based on the best compromise in performance for the network configuration. The vehicle optimization requires an algorithm that can accommodate the mixed-integer variables and non-linear analysis functions required to define the transportation network. As such, Simulated Annealing ${ }^{9}$ is chosen as the optimization algorithm for the vehicle optimization.

For the first seven city network, Atlanta is designated as the hub city and the optimization determines the vehicle design for an aircraft and the number of aircraft flying each route. The optimal cost for this network is $\$ 94,264$ and the design parameters are provided in Table 8 for the corresponding network configuration shown in Figure 6.

If we examine the solution depicted in Table 8 and Figure 6 we see that the range is defined to be higher than the range of Plane $\mathrm{A}$ in order to accommodate the distance requirements of the Albuquerque to Atlanta flight. The capacity is also set higher than that of Plane A in order to more accurately reflect the demand requirements of the network. Although there are still inefficiencies in the system design, the vehicle optimization produces a better architecture for the given network, as shown by a $12 \%$ reduction in cost.

For the largest seven city network, Chicago is designated as the hub city. Again, the vehicle design parameters and the number of aircraft flying each leg are optimized. The optimal cost for this network is $\$ 570,720$ and the design parameters are provided in Table 9 for the corresponding network configuration shown in Figure 7 .

If we examine Table 9 and Figure 7, we see that the range of the aircraft designed is between that of a Plane A design and a Plane B design. This compromise in range is due to the fact that transatlantic flights

$$
10 \text { of } 16
$$


Table 8. Aircraft Specifications for Vehicle Optimization for First Seven City Network

\begin{tabular}{|l|c|}
\hline Parameter & New Plane Design \\
\hline \hline Capacity $C(l b s)$ & 17,995 \\
\hline Range $R(n m i)$ & 1,558 \\
\hline Velocity $V_{c}(k t s)$ & 447 \\
\hline Wing Loading $W / S\left(l b / f t^{2}\right)$ & 105 \\
\hline Thrust to Weight $T / W$ & .31 \\
\hline Number of Engines $N_{\text {eng }}$ & 2 \\
\hline Fixed Cost $f(\$ /$ day $)$ & 3,421 \\
\hline Linear Cost $m(\$ / h r)$ & 1,251 \\
\hline
\end{tabular}

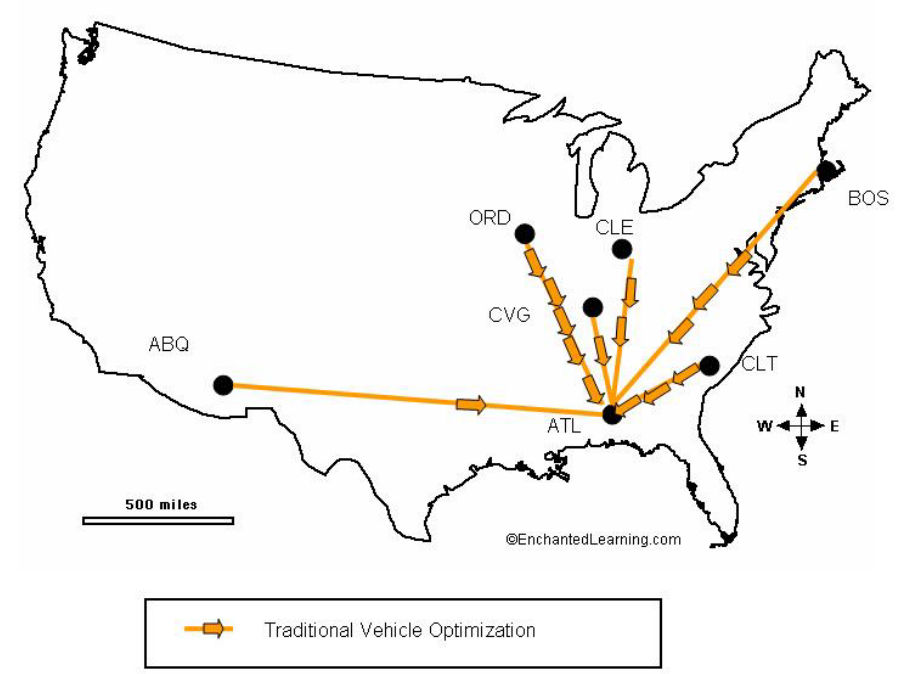

Figure 6. Vehicle Optimization Configuration for First Seven City Network

Table 9. Aircraft Specifications for Vehicle Optimization for Largest Seven City Network

\begin{tabular}{|l|c|}
\hline Parameter & New Plane Design \\
\hline \hline Capacity $C(l b s)$ & 128,050 \\
\hline Range $R(n m i)$ & 1,920 \\
\hline Velocity $V_{c}(k t s)$ & 540 \\
\hline Wing Loading $W / S\left(l b / f t^{2}\right)$ & 134 \\
\hline Thrust to Weight $T / W$ & .315 \\
\hline Number of Engines $N_{\text {eng }}$ & 2 \\
\hline Fixed Cost $f(\$ /$ day $)$ & 14,106 \\
\hline Linear Cost $m(\$ / h r)$ & 4,083 \\
\hline
\end{tabular}

11 of 16

American Institute of Aeronautics and Astronautics 


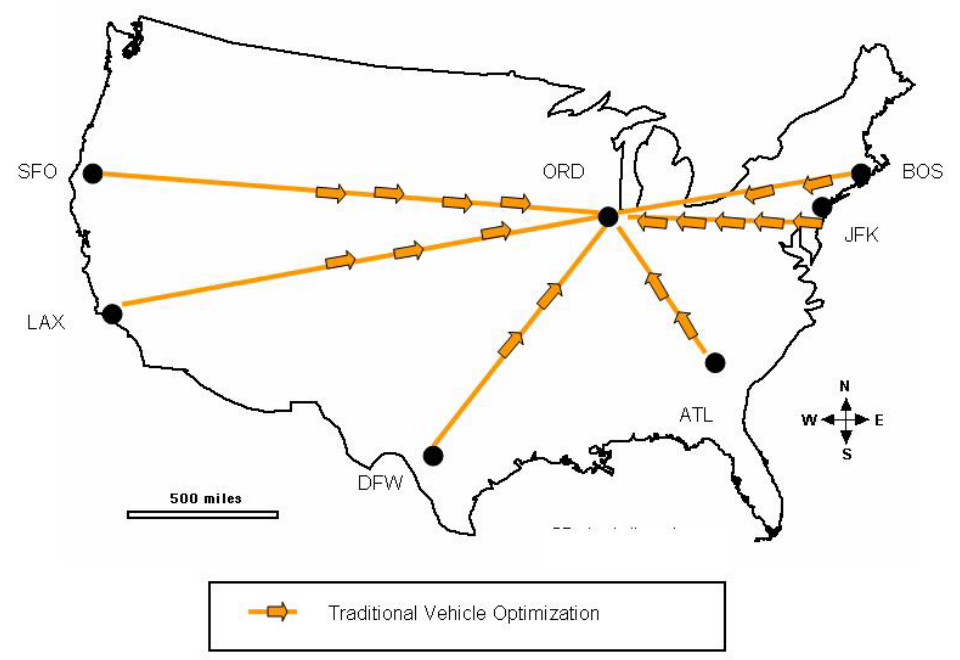

Figure 7. Vehicle Optimization Configuration for Largest Seven City Network

are not necessary for this architecture. The capacity of the aircraft designed by the vehicle optimization is between that of a Plane $\mathrm{B}$ and Plane $\mathrm{C}$, and reflects the large demand requirements for direct flights into Chicago. Although the vehicle is designed to reduce inefficiencies in the network, the requirement of only using direct flights actually increases the system cost by $10 \%$.

\section{Integrated Transportation Network Design Optimization}

For an integrated transportation network, the vehicle, network and operations definition are concurrently optimized. The design vector includes variables that define both the vehicle and network, and the system is subject to the constraints that govern the vehicle, network, and operations. The optimization algorithm chosen for the integrated transportation network design problem is Simulated Annealing, since the formulation consists of mixed-integer variables and non-linear analysis functions.

If we consider the design of a single vehicle and concurrently optimize the vehicle characteristics and the routes through the network for the first seven city network, the optimal transportation system design has a cost of $\$ 83,833$, which is a decrease in system cost of $22 \%$ over the traditional network optimization result and a decrease of $11 \%$ over the traditional vehicle optimization result. The vehicle design parameters for the integrated optimization are provided in Table 10 and the optimal configuration is shown in Figure 8 .

By analyzing the concurrently optimized design presented in Table 10 and Figure 8 we can see that a slightly larger aircraft, as compared to Plane A is designed to handle the distance requirements for both the Albuquerque-Atlanta and Albuquerque-Chicago flights and the demand requirements for more of the Chicago and Atlanta flights directly. However, since the concurrently optimized design is not constrained to fly only direct flights, the capacity of the aircraft is lower than that obtained by traditional vehicle optimization.

Analyzing the largest seven city network, the integrated transportation system design methodology produces an optimal system cost of $\$ 463,723$, which is a reduction in cost of $10 \%$ over the traditional network optimization and a reduction of $18 \%$ over the traditional vehicle optimization. The optimal vehicle design parameters for the integrated transportation design optimization are listed in Table 11 and the optimal configuration is provided in Figure 9 .

The concurrently optimized solution presented in Table 11 and Figure 9 is sized to be slightly smaller than Plane B. The reduction in range no longer accommodates the transatlantic flights from Boston, but does satisfy the distance requirements for the New York to Los Angeles and New York to San Francisco 


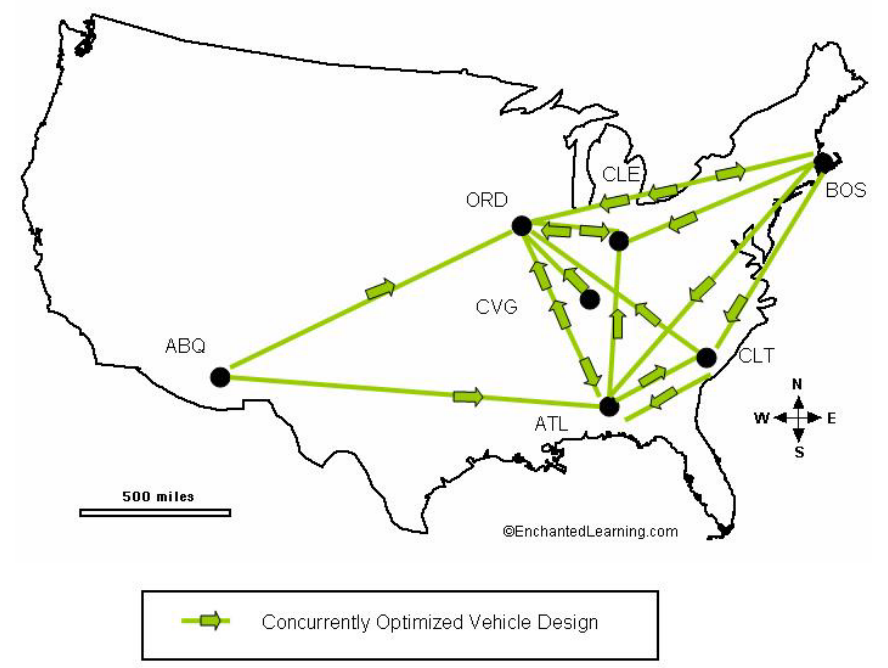

Figure 8. Optimal Configuration for Integrated Transportation System Design for First Seven City Network

Table 10. Aircraft Specifications for Integrated Transportation System Design for First Seven City Network

\begin{tabular}{|l|c|}
\hline Parameter & New Plane Design \\
\hline \hline Capacity $w(l b s)$ & 9,850 \\
\hline Range $r(n m i)$ & 1,253 \\
\hline Velocity $v(k t s)$ & 550 \\
\hline Wing Loading $W / S\left(l b / f t^{2}\right)$ & 105 \\
\hline Thrust to Weight $T / W$ & .302 \\
\hline Number of Engines $N_{\text {eng }}$ & 2 \\
\hline Fixed Cost $f(\$ /$ day $)$ & 2,320 \\
\hline Linear Cost $m(\$ / h r)$ & 986 \\
\hline
\end{tabular}

Table 11. Aircraft Specifications for Integrated Transportation System Design for Largest Seven City Network

\begin{tabular}{|l|c|}
\hline Parameter & New Plane Design \\
\hline \hline Capacity $w(l b s)$ & 69,884 \\
\hline Range $r(n m i)$ & 2,560 \\
\hline Velocity $v(k t s)$ & 550 \\
\hline Wing Loading $W / S\left(l b / f t^{2}\right)$ & 106 \\
\hline Thrust to Weight $T / W$ & .302 \\
\hline Number of Engines $N_{\text {eng }}$ & 2 \\
\hline Fixed Cost $f(\$ /$ day $)$ & 9,633 \\
\hline Linear Cost $m(\$ / h r)$ & 2,807 \\
\hline
\end{tabular}




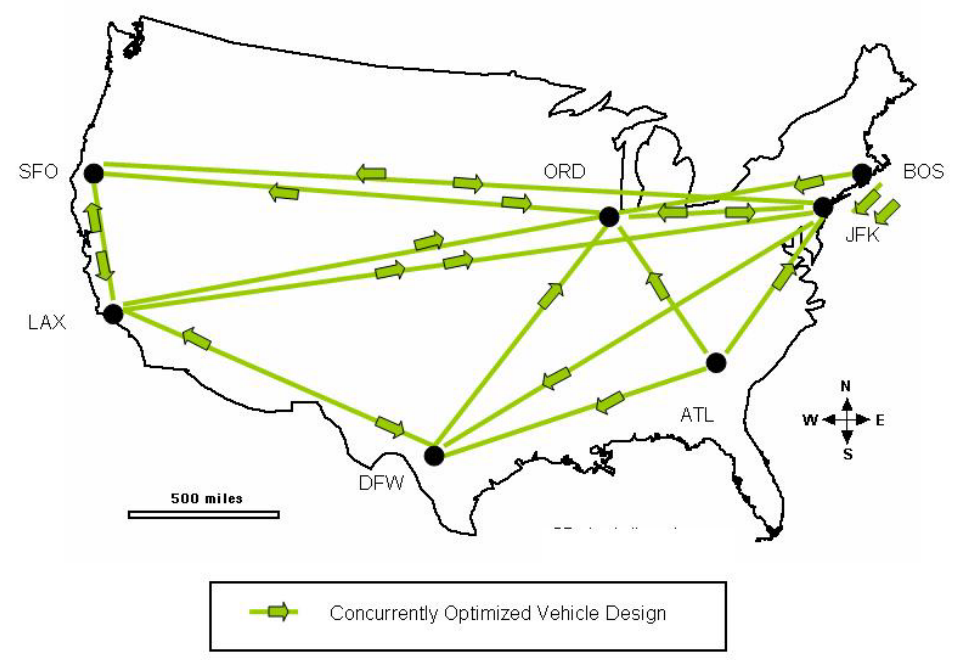

Figure 9. Optimal Configuration for Integrated Transportation System Design for Largest Seven City Network

flights. By reducing the range and the capacity of the vehicle design slightly, a reduction in aircraft costs is obtained and it is cheaper to utilize more of these aircrafts. Again, since direct flights are not required the capacity of the concurrently optimized solution is less than that of the vehicle optimization design, but has a greater range.

The integrated transportation system design methodology exploits the coupling of the vehicle and network by defining a more efficient set of operations for the transportation system. This effect can best be visualized by plotting the distance versus demand of each city in the network. In addition, vehicle design points are included by plotting the range versus capacity of the aircrafts. The effect of the vehicle designs can be interpreted as follows. All points lying within the bounding box of a vehicle design point can be fulfilled by a single direct flight of that vehicle. Any points to the right of the vehicle design point but below the upper bound of the box require at least one connection, as the distance exceeds the aircraft's range. Alternatively, any points above the vehicle design point but left of the right bound of the box require more than one flight as the demand exceeds the capacity of a single vehicle.

Figure 10 displays the distance and demand of the first seven city network and the vehicle design points for the pre-defined plane, Plane A, the traditional vehicle optimization design, and the integrated optimization design point. The other pre-defined vehicle design points are omitted for clarity as the design parameters of these planes exceed the distance and the demand of the network. The integrated optimization design point is only slightly right and above the Plane A design point; however, this difference allows the Albuquerque demand to be accommodated using a smaller and cheaper plane than Plane B. In addition, the Chicago to Cleveland and Chicago to Charlotte flights can be handled directly. By examining Figure 8, we see that only a single flight from Chicago to Charlotte is utilized. The Chicago to Cleveland route has a flight in each direction, however this is not a result of the Chicago to Cleveland demand, but demand from other cities into and out of Cleveland.

Figure 11 displays the distance and demand of the largest seven city network and the vehicle design points for the pre-defined planes, the traditional vehicle optimization design, and the integrated optimization design point. By examining Figure 11 we see that the integrated optimization design has a range that can handle the distance requirements of a New York to Los Angeles and New York to San Francisco flight, but the demand between these cities is almost twice the aircraft's capacity. If we examine Figure 9 we see that there are two flights from Los Angeles to New York and a direct flight in each direction from New York to San Francisco, which can accommodate the New York to Los Angeles and New York to San Francisco demand, 


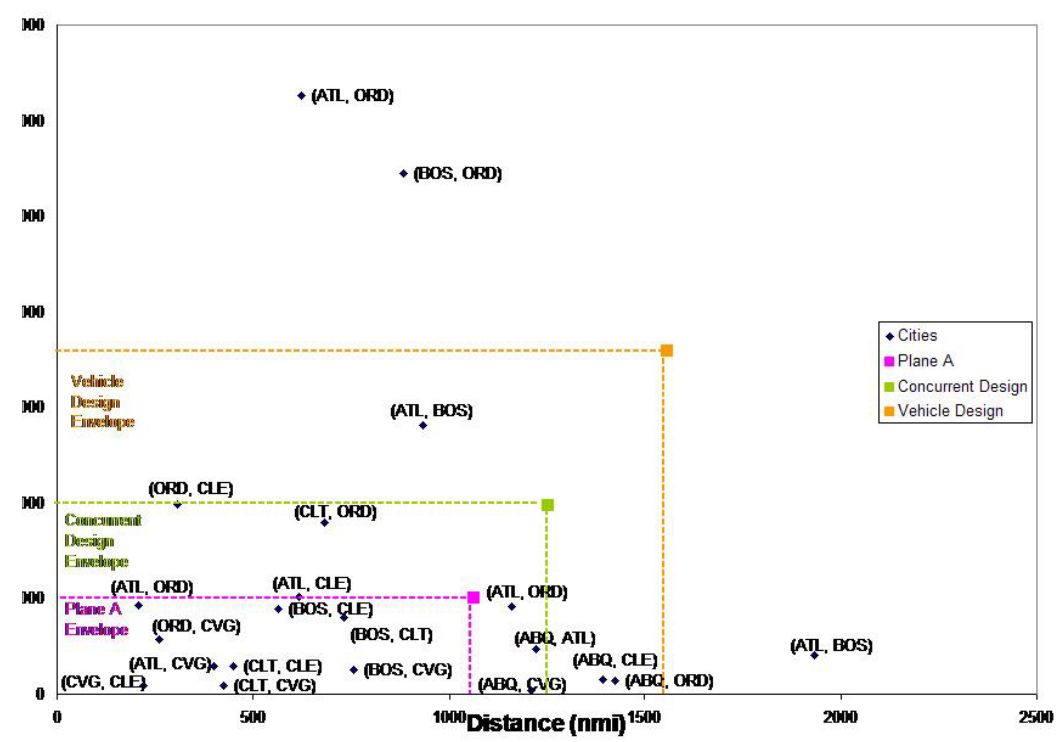

Figure 10. Distance versus Demand for First Seven City Network

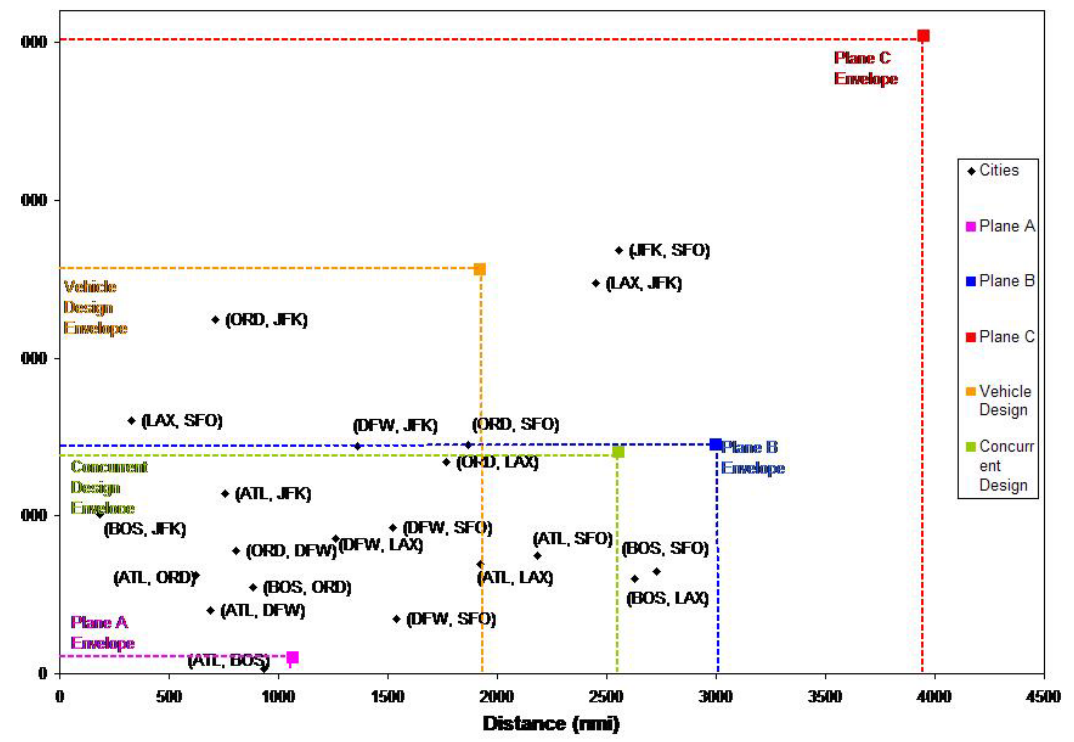

Figure 11. Distance versus Demand for Largest Seven City Network 
respectively. However, it is important to realize that some of the demand between these city pairs may be handled by other connecting flights as there is a Boston to New York flight that may require some of the Boston to Los Angles and Boston to San Francisco packages be flown on the return flights from LA and San Francisco, respectively.

\section{Conclusion}

In this paper, a methodology for integrated transportation network design was presented. By expanding the definition of a transportation system to include the vehicle definition as well as the network and operations during the design process, the system control volume was expanded to produce a systems level solution to the transportation architecture. The integrated transportation system design methodology provides an analysis of the different high-level sub-systems that comprise the transportation network and defines how the sub-systems interact. Utilizing the formulations developed to define the network, vehicle and operations, a concurrent optimization of the transportation system definition is obtained for two different example networks. The integrated transportation design methodology produced a minimum of a ten percent reduction in cost over traditional network optimization methods. In comparing the integrated design methodology for the two cases considered to traditional vehicle optimization, a minimum of an eleven percent reduction in cost was shown.

The results provided in this paper where obtained under highly restrictive modeling assumptions. For instance, the requirement that aircrafts fly only a single round trip route could be relaxed, allowing a single aircraft to visit multiple cities before returning to the original city; however this expansion would require tracking the flight times to ensure feasible connections. The demand provided for both networks was assumed to be fixed; however in reality, demand estimates are generally stochastic. This methodology could be extended to analyze probabilistic demand or analyze the effects of a demand evolution over time to define a robust transportation architecture. Finally, this paper assumed no costs for ground facility operations, which again is unrealistic. By expanding the system definition further to track ground operations, a better transportation architecture could be defined.

The value of this analysis is not in the actual results obtained but in the problem formulation. By expanding the definition of the system to include the vehicle, network and operations design a more efficient system architecture can be obtained that reduces operating costs. Ideally, this methodology would aid in strategic planning or investments at a major cargo airline or provide insight about market need to aircraft designers.

\section{References}

${ }^{1}$ Maier, M., "Architecting Principles for System-of-Systems," Systems Engineering, Vol. 1, No. 4, 1998.

${ }^{2}$ Yang, L. and Kornfeld, R., "Examiniation of the Hub-and-Spoke Network: A Case Example Using Overnight Package Delivery," 41st Aerospace Sciences Meeting and Exhibit, AIAA, 2003.

${ }^{3}$ Ravindra Ahuja, T. M. and Orlin, J., Network Flows: Theory, Algorithms and Applications, Prentice Hall, 1993.

${ }^{4}$ David Simchi-Levi, Julien Bramel, X. C., The Logic of Logistics: Theory, Algorithms, and Applications for Logistics and Supply Chain Management, Springer, 2005.

${ }^{5}$ Barnhart, C., e. a., "Flight String Model for Aircraft Fleeting and Routing," Transportation Science, Vol. 32, 1998.

${ }^{6}$ William Crossley, M. M. and Nusawardhana, "Variable Resource Allocation Using Multidisciplinary Optimization: Initial Investigations for System of Systems," 10th AIAA-ISSMO Multidisciplinary Analysis and Optimization Conference, AIAA, 2004 .

${ }^{7}$ Meissinger, H. and Collins, J., "Mission Design and System Requirements for a Multiple-Function Orbital Transfer Vehicle," AIAA Space Technology Conference, No. 99-42028, 1999.

${ }^{8}$ Raymer, D. P., Aircraft Design: A Conceptual Approach, 3rd edition, AIAA Educational Series, 1999.

${ }^{9}$ S. Kirkpatrick, C. D. Gelatt, M. P. V., "Optimization by Simulated Annealing," Science, Vol. 220, 4598, 1983, pp. 671-680. 